Check for updates

Cite this: RSC Adv., 2017, 7, 46576

Received 27th July 2017

Accepted 26th September 2017

DOI: $10.1039 / \mathrm{c} 7 \mathrm{ra0} 8282 \mathrm{~g}$

rsc.li/rsc-advances

\section{Morphological control of poly(vinylidene fluoride) alayered double hydroxide composite fibers using metal salt anions and their enhanced performance for dye removal $\dagger$}

\begin{abstract}
Qingqing Mei, Weiyang Lv, Miao Du (DD * and Qiang Zheng
Self-standing membranes of poly(vinylidene fluoride) alayered double hydroxide (PVDF(ALDH) composite fibers were fabricated by using metal salts with different anions $\left(\mathrm{NO}_{3}{ }^{-}, \mathrm{Cl}^{-}, \mathrm{SO}_{4}{ }^{2-}\right)$ as the reactants through a combination of electrospinning and hydrothermal treatment. The anions have a significant effect on the morphology and loading amount of LDHs anchored onto the surface of PVDF nanofibers. In a monovalent anion (such as nitrate) system, small crystal nuclei were formed. Only a small proportion with a large size grew up to well-defined hexagonal LDH layered nanocrystals with large thicknesses. However in a divalent anion sulfate system, large crystal nuclei were generated first and most of them could grow up to thin and curly LDH layered nanocrystals owing to the serious steric hindrance. Welldefined PVDFaCoAl-LDH composite fibers with a hierarchical structure and uniform core/sheath morphology could only be obtained in the sulfate system, which could act as an excellent adsorbent for treating dye solutions. The obtained PVDFCCOAl-LDH core/sheath fibers present a maximum adsorption capacity of $621.17 \mathrm{mg} \mathrm{g}^{-1}$ for methyl orange (MO). Moreover, benefiting from the perfect properties and stable form, the composite fiber membrane could be used directly in membrane filtration with high removal efficiency and cycling stability. The MO removal rate of the membrane remained above $93 \%$ with a maximum flux of $140 \mathrm{~L}\left(\mathrm{~m}^{2} \mathrm{~h}\right)^{-1}$ despite three times of regeneration, indicating its practical application for the efficient treatment of dye pollution.
\end{abstract}

\section{Introduction}

Dyes are basic chemical compounds that impart color and are widely used in many industries, such as textile, paper, leather tanning, and plastics. ${ }^{1}$ More than 100000 dyes are available commercially, and over one million tons of dyes are produced per year. ${ }^{2}$ However, about $10-15 \%$ of dyes are discharged in wastewater during manufacturing or processing, thereby causing serious water pollution problems because many dyes are toxic, carcinogenic, and mutagenic even in low concentrations. ${ }^{3}$ Various methods, such as coagulation, ${ }^{4}$ degradation, ${ }^{5}$ adsorption, ${ }^{6}$ oxidation ${ }^{7}$ and membrane filtration, ${ }^{8}$ have been developed to remove dyes from contaminated water. Among them, adsorption is one of the most effective techniques because it is economically feasible, easy to perform, and insensitive to toxic substances. ${ }^{1,3}$ Although numerous materials

MOE Key Laboratory of Macromolecular Synthesis and Functionalization, Key Laboratory of Adsorption and Separation Materials \& Technologies of Zhejiang Province, Department of Polymer Science and Engineering, Zhejiang University, Hangzhou, 310027, China. E-mail: dumiao@zju.edu.cn; Fax: +86-571-87953075; Tel: +86-571-87953075

$\dagger$ Electronic supplementary information (ESI) available. See DOI: 10.1039/c7ra08282g have been used as adsorbents, almost none meets the high level industry requirements. Therefore, novel, highly efficient adsorbents with large-scale preparation and easy retrievability should be developed. ${ }^{9}$

Layered double hydroxides (LDHs) are a class of layered anionic clays usually described by a general formula of $\left[\mathrm{M}_{1-x}{ }^{2+} \mathrm{M}_{x}^{3+}(\mathrm{OH})_{2}\right]\left(\mathrm{A}^{n-}\right)_{x / n} m \mathrm{H}_{2} \mathrm{O}$, where $\mathrm{M}^{2+}$ and $\mathrm{M}^{3+}$ are the divalent and trivalent metal ions, respectively, in positively charged layers and $\mathrm{A}^{n-}$ is the interlayer anion. ${ }^{10}$ LDHs have received much attention in the past decades as a promising, highly efficient adsorbent for wastewater treatment because of their large specific surface area, high charge density, and superior interlayer anion exchange property. ${ }^{11}$ The composition (kinds of $\mathrm{M}^{2+}$ or $\mathrm{M}^{3+},{ }^{12}$ ratio of $\mathrm{M}^{2+} / \mathrm{M}^{3+},{ }^{13}$ interlayer anions ${ }^{14}$ ), morphology ${ }^{15}$ or architecture ${ }^{9,16}$ of LDHs with different specific surface areas remarkably influences their adsorption performance. Furthermore, LDHs is commonly obtained in powder state, which is not convenient to operate and reuse and simultaneously causes the loss of adsorbents and secondary pollution for the treated water. Therefore, a growth template with a 3D shape and high-specific surface area is needed to obtain a self-standing adsorbent.

Electrospinning is an efficient and simple method to fabricate fibers down to the micro- and nanometer scale. Electrospun 
fibers possess high porosity, large specific surface area, and facile modification properties and are widely used as matrix or template to fabricate multilevel micro- and nanostructure materials for environmental remediation. ${ }^{17-19}$ Liu et al. prepared hierarchical $\mathrm{SiO}_{2} @ \gamma-\mathrm{AlOOH}$ (boehmite) core/sheath fibers through the hydrothermal growth of boehmite on the surface of electrospun $\mathrm{SiO}_{2}$ fibers; this material showed an enhanced removal efficiency for organic dye and bacteria. ${ }^{20}$ Hota et al. reported a self-standing membrane of polyacrylonitrile (PAN)/iron oxide nanocomposite fibers via fabricating electrospun PAN, followed by the hydrothermal treatment for in situ growth of iron oxide nanoparticles; this material possessed a maximum adsorption capacity of $52.08 \mathrm{mg} \mathrm{g}^{-1}$ for Congo red dye. ${ }^{21}$

Given the high temperature and high pressure in the hydrothermal synthesis of LDHs, poly(vinylidene fluoride) (PVDF) electrospun nanofibers were often selected as a growth template with the advantages of flexibility, superior mechanical strength and outstanding thermal stability. ${ }^{22}$ Although Sailaja et al. have successfully grown LDH layered nanocrystals on the surface of PVDF electrospun substrate acting as an anion conducting membrane, its morphological tunability remains unclear. $^{23}$ Here, we fabricated several forms of self-standing PVDF@LDH composite fibers with LDH layered nanocrystals anchored on the surface of PVDF nanofibers through different hydrothermal treatment conditions. Synthesis conditions and metal salts are the two key factors for the morphological control of final products in a hydrothermal urea hydrolysis system. ${ }^{24}$ Optimal synthesis conditions, such as reaction time, reaction temperature, and proportion and concentration of the precursor solution, could be easily determined through some exploring experiments. For the metal salt, the influence of metal cations on the properties of LDHs has been widely investigated. ${ }^{25}$ However, few studies focused on the anion species because most researchers thought that the metal salt anions were mainly involved in the late stage of the LDHs formation, and not the main body structure of LDH layered nanocrystals. ${ }^{26,27}$ In the present study, various anion species were used during the growth of LDHs on the surface of electrospun nanofiber membrane, and the anion species were found to play a key role in the morphological control of PVDF@LDH composite nanofibers. Among these species, the uniform core/sheath structure was obtained only when divalent and trivalent metal sulfates were used as the reactants. These as-obtained PVDF@CoAl-LDH core/sheath fibers could be used in methyl orange removal. The adsorption kinetics and isotherms results showed that this novel adsorbent presented excellent adsorption property, and the adsorption was driven by the interlayer anion exchange and the external surface attraction. Benefiting from the hierarchical porous structure and stable form of the film, the fibrous membrane with high removal efficiency and excellent recycling stability could be used directly in filtration.

\section{Experimental}

\section{Materials and reagents}

PVDF $\left(M_{\mathrm{w}}: 53000\right)$ was obtained from Sigma-Aldrich. Cobalt sulfate heptahydrate $\left(\mathrm{CoSO}_{4} \cdot 7 \mathrm{H}_{2} \mathrm{O}\right)$, cobalt nitrate hexahydrate
$\left(\mathrm{Co}\left(\mathrm{NO}_{3}\right)_{2} \cdot 6 \mathrm{H}_{2} \mathrm{O}\right)$, magnesium nitrate hexahydrate $\left(\mathrm{Mg}\left(\mathrm{NO}_{3}\right)_{2}{ }^{-}\right.$ $\left.\cdot 6 \mathrm{H}_{2} \mathrm{O}\right)$, magnesium chloride hexahydrate $\left(\mathrm{MgCl}_{2} \cdot 6 \mathrm{H}_{2} \mathrm{O}\right)$, cobalt chloride hexahydrate $\left(\mathrm{CoCl}_{2} \cdot 6 \mathrm{H}_{2} \mathrm{O}\right)$, aluminum chloride nonahydrate $\left(\mathrm{AlCl}_{3} \cdot 9 \mathrm{H}_{2} \mathrm{O}\right)$, aluminum sulfate octadecahydrate $\left(\mathrm{Al}_{2}\left(\mathrm{SO}_{4}\right)_{3} \cdot 18 \mathrm{H}_{2} \mathrm{O}\right)$, and methyl orange (MO, 96\% purity) were purchased from Aladdin reagent. Magnesium sulfate heptahydrate $\left(\mathrm{MgSO}_{4} \cdot 7 \mathrm{H}_{2} \mathrm{O}\right)$, aluminum nitrate nonahydrate $\left(\mathrm{Al}\left(\mathrm{NO}_{3}\right)_{3} \cdot 9 \mathrm{H}_{2} \mathrm{O}\right)$, urea, $N, N$-dimethyl formamide (DMF), acetone (ACE), and sodium sulfate $\left(\mathrm{Na}_{2} \mathrm{SO}_{4}\right)$ were all supplied by Sinopharm Chemical Reagent Co., Ltd., China. All the reagents were used as received without further purification. Deionized water was used in the experiments.

\section{Preparation of PVDF electrospun nanofibers}

PVDF was dissolved in binary mixed solvent of DMF/ACE with volume ratio of $6: 4$ under magnetic stirring at room temperature, and the transparent solution with PVDF concentration of $15 \mathrm{wt} \%$ was transferred into a plastic syringe for electrospinning. The solution was fed at a needle diameter of $0.7 \mathrm{~mm}$ and constant speed of $0.5 \mathrm{~mL} \mathrm{~h}^{-1}$, with an applied current potential of $15 \mathrm{kV}$. The distance between the needle tip and the collecting aluminum foil was $13 \mathrm{~cm}$, and the fibrous membrane was peeled off and dried under vacuum at $130{ }^{\circ} \mathrm{C}$ for $5 \mathrm{~h}$. Morphology and structure of PVDF electrospun fibers could be adjusted by varying the processing parameters, ${ }^{\mathbf{2 8}}$ and the optimal processing parameters described above were chosen through exploring experiments that have been discussed detailedly in ESI. $\dagger$

\section{Preparation of PVDF@LDH composite fibers}

The PVDF fibrous membrane $\left(3.0 \times 3.0 \mathrm{~cm}^{2}, \sim 6 \mathrm{mg}\right)$ was pretreated by soaking in ethanol and immersing in a precursor solution consisting of a certain amount of $\mathrm{CoSO}_{4} \cdot 7 \mathrm{H}_{2} \mathrm{O}$ $\left(40 \mathrm{mmol} \mathrm{L}^{-1}\right), \mathrm{Al}_{2}\left(\mathrm{SO}_{4}\right)_{3} \cdot 18 \mathrm{H}_{2} \mathrm{O}$ and urea, which was dissolved in $50 \mathrm{~mL}$ of deionized water with carbon dioxide removed at molar ratio of $\mathrm{Co} / \mathrm{Al}=2$ and urea/ $\left[\mathrm{SO}_{4}{ }^{2-}\right]=8$. The membrane was subsequently transferred into a $100 \mathrm{~mL}$ Teflon-lined stainless steel autoclave that was sealed, heated, and kept at $105{ }^{\circ} \mathrm{C}$ for $12 \mathrm{~h}$. After being cooled down to room temperature, the obtained products were thoroughly washed with water, dried at $60{ }^{\circ} \mathrm{C}$ for $2 \mathrm{~h}$, and designated as PVDF@CoAl-LDH core/ sheath fibers. Other PVDF@LDH composite fibers were synthesized in the same manner; for example, $\mathrm{MgSO}_{4} \cdot 7 \mathrm{H}_{2} \mathrm{O}$ and $\mathrm{Al}_{2}\left(\mathrm{SO}_{4}\right)_{3} \cdot 18 \mathrm{H}_{2} \mathrm{O}$ were the reactants used in $\mathrm{MgAl}$-sulfate system and were denoted as $\operatorname{MgAl}\left(\mathrm{SO}_{4}\right)$. In addition, the $\mathrm{LDH}$ powder that remained in the reaction solution was also collected through filtration and was vacuum freeze-dried for characterization.

\section{Characterization}

X-ray diffraction (XRD) patterns of the samples were conducted on a Rigaku D/max 2550 diffractometer (Shimadzu Corporation, Kyoto, Japan) with $\mathrm{Cu} \mathrm{k} \alpha$ radiation $(\lambda=0.1542 \mathrm{~nm})$ under a tension of $40 \mathrm{kV}$ and a current of $30 \mathrm{~mA}$. The morphology and surface elementary composition were investigated by field emission scanning electron microscopy and energy dispersive 
X-ray (FESEM-EDX) (Hitachi S-4800, Japan). Fourier transform infrared spectrum (FT-IR) of LDH powder was recorded in the range of $400-4000 \mathrm{~cm}^{-1}$ on Vector 22 (Bruker, Germany). Attenuated total reflectance infrared spectrum (ATR-IR) of PVDF nanofibers and PVDF@CoAl-LDH core/sheath fibers was recorded on Nicolet 6700 (Thermo Fisher Scientific LLC) in the same range. $\mathrm{N}_{2}$ adsorption-desorption isotherms were measured with a Quantachrome Autosorb-1-c system. The surface modification was investigated by X-ray photoelectron spectroscopy (XPS). Thermogravimetric analysis (TGA) (Q50, TA Instruments, USA) was used to analyze the content of LDHs on the composite fibers. These samples were heated from $50{ }^{\circ} \mathrm{C}$ to $800{ }^{\circ} \mathrm{C}$ at a rate of $10{ }^{\circ} \mathrm{C} \min ^{-1}$ under a nitrogen atmosphere. Contact angles (CAs) were measured by an optical CA measuring device (HarkeSPCA, Peking Harke Experimental Instrument Factory, China).

\section{Measurements of adsorption performance}

Given its unique structure and wide application, MO was selected as the typical anionic dye to determine the adsorption properties of the as-obtained PVDF@CoAl-LDH core/sheath fibers. The equilibrium adsorption experiments were performed as follows: about $4 \mathrm{mg}$ of PVDF@CoAl-LDH core/sheath fibers were immersed in $10 \mathrm{~mL}$ of $\mathrm{MO}$ aqueous solution with an initial concentration ranging from $20 \mathrm{mg} \mathrm{L}^{-1}$ to $500 \mathrm{mg} \mathrm{L}^{-1}$. The oscillation treatment was conducted at $200 \mathrm{rpm}$ and $30{ }^{\circ} \mathrm{C}$ for $7 \mathrm{~h}$ followed by standing for about 3 days. The concentration of MO that remained in the solution was analyzed by using a Lambda $35 \mathrm{UV}$-vis adsorption spectrometer at a wavelength of $465 \mathrm{~nm}$. MO concentration of $200 \mathrm{mg} \mathrm{L}^{-1}$ was used in the adsorption kinetics experiment while other conditions were kept similar to those in the equilibrium adsorption test. The $\mathrm{pH}$ value of the MO solution in above experiments was kept at 7.0. To explore the influence of $\mathrm{pH}$ on the adsorption performance, the MO solution $\mathrm{pH}$ value was adjusted by using $0.1 \mathrm{~mol} \mathrm{~L}^{-1}$ $\mathrm{HCl}$ and $0.1 \mathrm{~mol} \mathrm{~L}^{-1} \mathrm{NaOH}$. The adsorbed amount of $\mathrm{MO}(Q, \mathrm{mg}$ $\mathrm{g}^{-1}$ ) was calculated from eqn (1),

$$
Q=\frac{\left(C_{0}-C_{\mathrm{r}}\right) V}{m}
$$

where $C_{0}$ and $C_{\mathrm{r}}$ are the MO concentration $\left(\mathrm{mg} \mathrm{L}^{-1}\right)$ in the initial and the treated solutions, respectively; $V$ is the volume of the solution (L); and $m$ is the mass of PVDF@CoAl-LDH core/sheath fibers $(\mathrm{g})$.

\section{Membrane filtration of the MO solution}

The experiment was carried out in a homemade dead-end filtration apparatus, where five pieces of PVDF@CoAl-LDH core/sheath fibrous membrane with an effective diameter of $2.0 \mathrm{~cm}$ were used. The MO aqueous solution with a concentration of $10 \mathrm{mg} \mathrm{L}^{-1}$ was filtered through the membrane with the height of the liquid column of $13.0 \mathrm{~cm}$ simply by gravity. Filtrate was collected at a certain time interval to detect the concentration of the remaining MO. The flux of the MO solution $(F, \mathrm{~L}$ $\left.\mathrm{m}^{-2} \mathrm{~h}^{-1}\right)$ and the removal efficiency $(R)$ can be calculated using eqn (2) and (3), respectively,

$$
\begin{gathered}
F=\frac{\Delta V}{A \Delta t} \\
R=\left(1-\frac{c_{\mathrm{p}}}{c_{\mathrm{f}}}\right) \times 100 \%
\end{gathered}
$$

where $\Delta V$ represents the volume of filtrate (L) collected within one time interval $\Delta t(\mathrm{~h}), A$ is the effective membrane area $\left(\mathrm{m}^{2}\right)$, $c_{\mathrm{p}}$ and $c_{\mathrm{f}}$ are the MO concentrations in the permeation and feed solution $\left(\mathrm{mg} \mathrm{L}^{-1}\right)$ respectively.

In the regeneration and reuse processes, the spent membrane was removed out and immersed in a high concentration of $\mathrm{Na}_{2} \mathrm{SO}_{4}$ solution $\left(0.5 \mathrm{~mol} \mathrm{~L}{ }^{-1}, 50 \mathrm{~mL}\right)$ and then oscillated at $200 \mathrm{rpm}$ and $30{ }^{\circ} \mathrm{C}$ for $0.5 \mathrm{~h}$ to desorb $\mathrm{MO}$ anions. Desorption was repeated three times, and the $\mathrm{Na}_{2} \mathrm{SO}_{4}$ solution was replaced with deionized water for another three times. Afterwards, the membrane was ready for the next run.

\section{Results and discussion}

\section{Effect of metal salt anions on LDH morphology}

Taking MgAl-LDH as an example, three kinds of anions $\left(\mathrm{NO}_{3}{ }^{-}\right.$, $\mathrm{Cl}^{-}, \mathrm{SO}_{4}{ }^{2-}$ ) were selected for the synthesis while the other experimental conditions (salt molar concentration, reaction time, and temperature) were kept constant. The as-obtained PVDF@LDH composite fibers exhibit a distinct architecture structure. When nitrate or chloride was used, well-defined hexagonal LDH layered nanocrystals were sparsely anchored on the surface of PVDF nanofibers or passed through them with a residual large area of bare fiber surface (Fig. 1a and b). However, when sulfate was used (Fig. 1c), the LDH layered nanocrystals in a form of cluster were grown densely on the surface of PVDF nanofibers, and hardly any bare fibers could be observed. Thus, PVDF@MgAl-LDH core/sheath fibers with curly LDH layered nanocrystals were obtained. When the metal cations were changed to $\mathrm{Co}^{2+}$ and $\mathrm{Al}^{3+}$, that is, the CoAl-LDH system, similar phenomena were observed (Fig. 1d-f). In the case of using $\mathrm{NO}_{3}{ }^{-}, \mathrm{Cl}^{-}$as anions, CoAl-LDH layered nanocrystals were distributed sparsely on the surface of the PVDF nanofibers, whereas densely grown layered nanocrystals and PVDF@CoAl-LDH core/sheath fibers with well-defined hybrid fiber structure were obtained in the sulfate system. This finding further confirmed the importance of anion species on the morphological control of PVDF@LDH composites. The morphological time evolution of PVDF@LDH composite fibers synthesized by using $\operatorname{MgAl}\left(\mathrm{SO}_{4}\right)$ and $\operatorname{MgAl}\left(\mathrm{NO}_{3}\right)$ was further explored to analyze the influence of anions.

\section{Time evolution of PVDF@LDH composite fibers with different metal salt anions}

Fig. 2 presents the morphological time evolution of PVDF@LDH composite fibers with $\mathrm{MgAl}\left(\mathrm{SO}_{4}\right)$ and $\mathrm{MgAl}\left(\mathrm{NO}_{3}\right)$ as the reactants, respectively. For sulfate (Fig. 2a-d), when the reaction time $(t)$ was $3 \mathrm{~h}$, some irregular blobs with a diameter of 100$200 \mathrm{~nm}$ appeared densely on the surface of the PVDF fibers. The EDX analysis of the intermediate products at $3 \mathrm{~h}$ as shown in Table 1 exhibits that the $\mathrm{Al} / \mathrm{Mg}$ ratio is about 42.5 for sulfate 

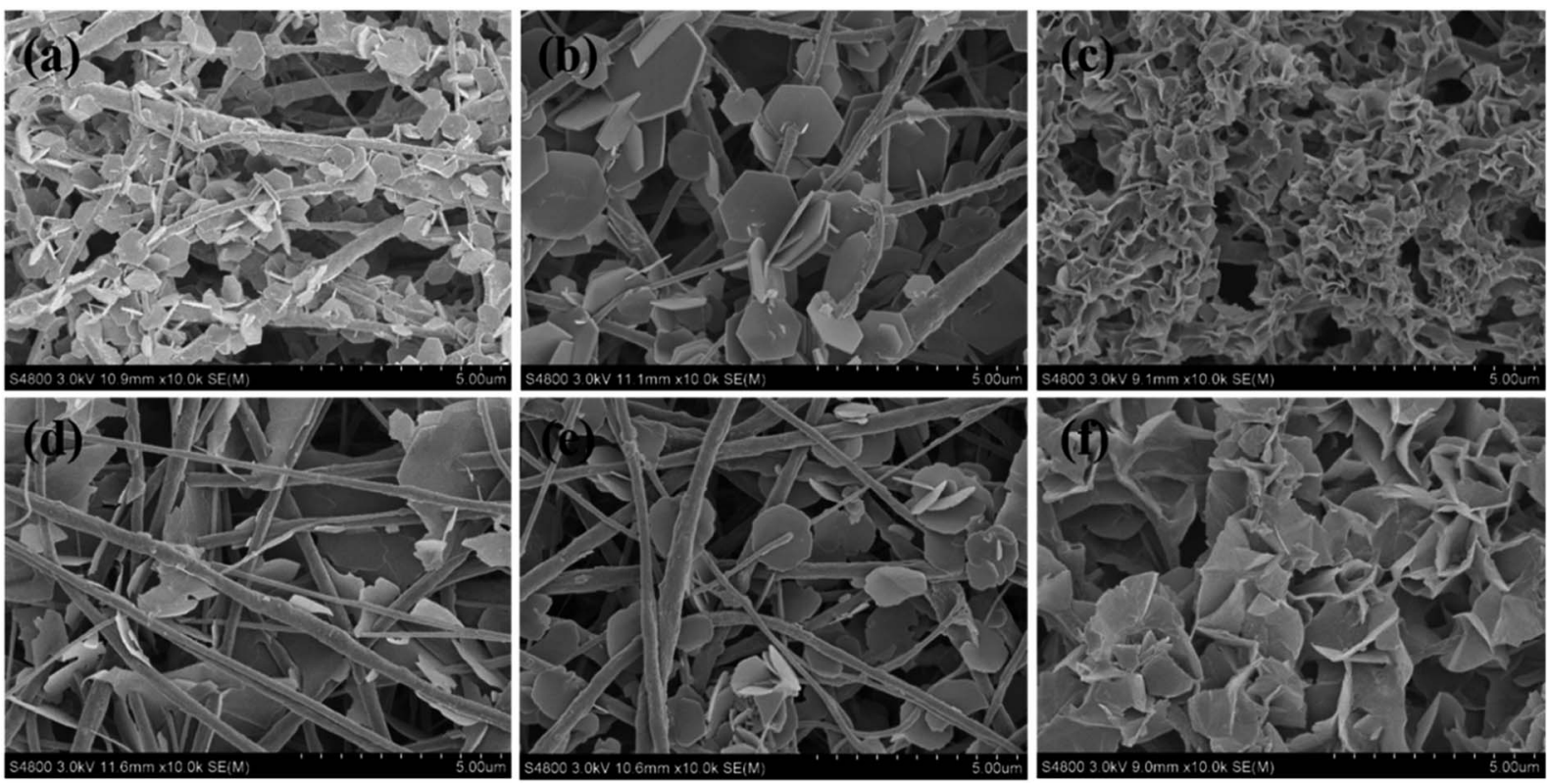

Fig. 1 FESEM pictures of PVDFaLDH composite fibers synthesized by different metal salts: (a) $\mathrm{MgAl}\left(\mathrm{NO}_{3}\right)$; (b) $\mathrm{MgAl}(\mathrm{Cl})$; (c) $\left.\mathrm{MgAl}_{(\mathrm{SO}}\right)_{4}$; (d) $\mathrm{CoAl}\left(\mathrm{NO}_{3}\right)$; (e) $\mathrm{CoAl}(\mathrm{Cl})$; (f) $\mathrm{CoAl}\left(\mathrm{SO}_{4}\right)$ with the total metal concentration $=60 \mathrm{mmol} \mathrm{L}{ }^{-1}$, reaction time $=12 \mathrm{~h}$, temperature $=105{ }^{\circ} \mathrm{C}$.
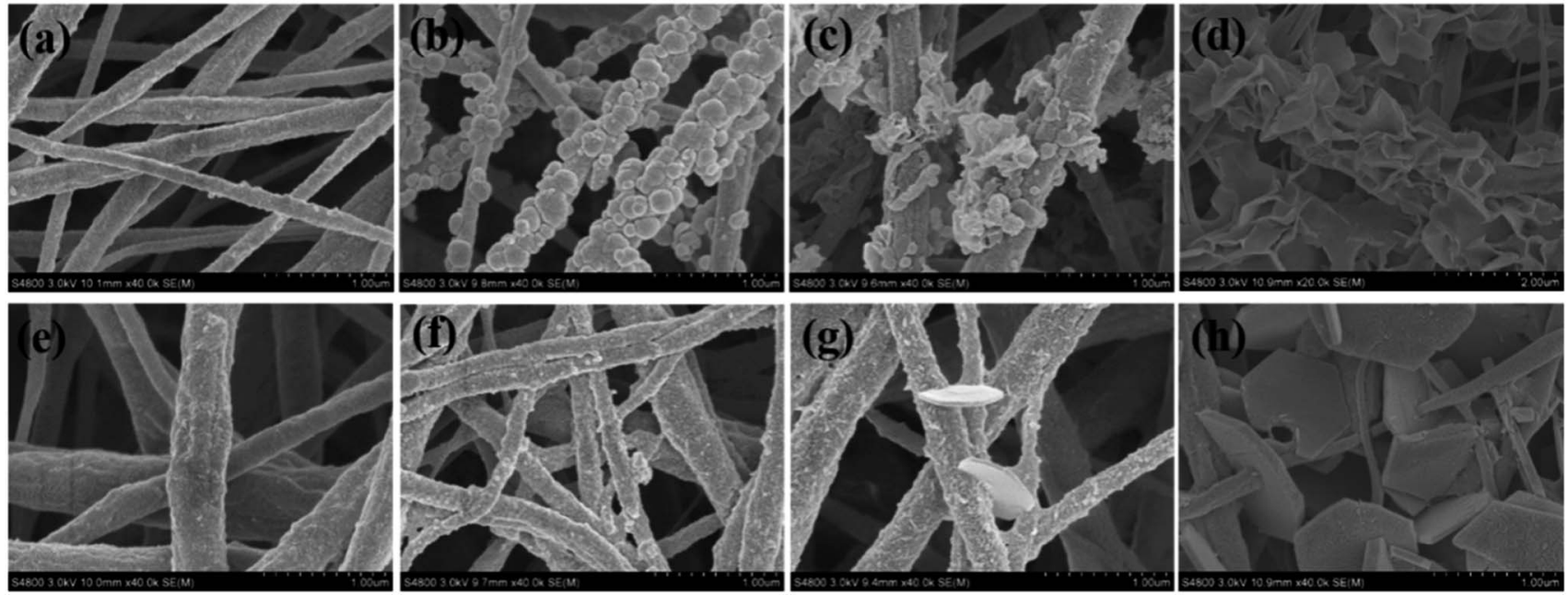

Fig. 2 FESEM pictures of PVDF(aLDH composite fibers synthesized by (a-d) MgAl( $\left.\mathrm{SO}_{4}\right)$ and (e-h) MgAl( $\left.\mathrm{NO}_{3}\right)$ with different reaction times: (a, e) $1.5 \mathrm{~h}$; (b, f) $3 \mathrm{~h}$; (c, g) $6 \mathrm{~h}$; (d, h) $24 \mathrm{~h}$.

Table 1 The EDX element composition analysis (at\%) of the surface of PVDFaLDH composite fibers synthesized by $\mathrm{MgAl}\left(\mathrm{SO}_{4}\right)$ and $\mathrm{MgAl}\left(\mathrm{NO}_{3}\right)$ with different reaction times

\begin{tabular}{lllllllll}
\hline & & \multicolumn{2}{c}{ Element } & & & & & \\
\cline { 3 - 8 } Sample & & $\mathrm{C}$ & $\mathrm{F}$ & $\mathrm{Mg}$ & $\mathrm{Al}$ & $\mathrm{O}$ & $\mathrm{N}$ & $\mathrm{S}$ \\
\hline $\operatorname{MgAl}\left(\mathrm{SO}_{4}\right)$ & $1.5 \mathrm{~h}$ & 74.11 & 22.85 & 0.03 & 0.00 & 1.90 & 0.93 & 0.18 \\
& $3 \mathrm{~h}$ & 59.52 & 26.25 & 0.06 & 2.55 & 7.69 & 3.05 & 0.48 \\
$\operatorname{MgAl}\left(\mathrm{NO}_{3}\right)$ & $1.5 \mathrm{~h}$ & 69.02 & 27.20 & 0.13 & 0.13 & 1.86 & 1.68 & 0.00 \\
& $3 \mathrm{~h}$ & 61.34 & 29.58 & 0.18 & 1.38 & 3.68 & 3.83 & 0.00
\end{tabular}

system. Based on current findings and previous reports, ${ }^{29}$ these blobs should be amorphous colloidal aluminum-rich hydroxide as the nucleus. When the reaction time increased, LDH crystals grew, and clusters of LDH layered nanocrystals were formed, which gradually covered the fiber surface.

For the nitrate system (Fig. 2e-h), the small aluminum-rich hydroxide intermediate product (the $\mathrm{Al} / \mathrm{Mg}$ ratio is about 7.67) that appeared on the surface was an irregular particle at $t=3 \mathrm{~h}$ and smaller than that in the sulfate system at the same reaction time. Only a few LDH layered nanocrystals were observed on the fiber surface at $t=6 \mathrm{~h}$. With the reaction time increased further, well-defined hexagonal LDH layered nanocrystals with the length of $\sim 1 \mu \mathrm{m}$ and the thickness of $\sim 100 \mathrm{~nm}$ were anchored 
or passed through the PVDF fibers. The $\mathrm{Al} / \mathrm{Mg}$ ratio at $t=3 \mathrm{~h}$ for both sulfate and nitrate systems is larger than the stoichiometric ratio of $\mathrm{Al} / \mathrm{Mg}$ in the initial reaction solution, i.e. $1: 2$, indicating that both the nuclei are authentically amorphous colloidal aluminum-rich hydroxide though the size of the nucleus for the sulfate system is larger than that in the nitrate system.

The LDH powder in the solution at different reaction times was also collected and characterized (Fig. 3). Similar to those on the surface of PVDF nanofibers, relatively large aluminum-rich hydroxide particles were observed in the sulfate system at $t=$ $3 \mathrm{~h}$, and curly and thin LDH layered nanocrystals were developed at $t=12 \mathrm{~h}$. However, in the nitrate system, small aluminum-rich hydroxide particles were generated at $t=3 \mathrm{~h}$, and thick and well-defined hexagonal LDH layered nanocrystals were grown. These results revealed that the growth of $\mathrm{LDH}$ layered nanocrystals shared the same evolution mechanism and process on the PVDF nanofiber surface and in the solution, implying that the effect of anion species on the $\mathrm{LDH}$ morphology was not induced by the surface character of PVDF fibers.

The total LDH amount obtained (including the LDHs both in the solution and on the surface of nanofibers) was almost similar within the same reaction time for the sulfate and nitrate systems. This finding indicates that the anion species of metal salts may affect the LDH morphology but not the reaction yield.

\section{Growth mechanism of LDHs with various metal salt anions}

The nucleation and growth of LDH crystals in the solution are identical to those on the PVDF nanofiber surface because the interaction between LDHs and PVDF is weak, which has been explained further in ESI. $\dagger$ The LDH morphology distinction between sulfate and nitrate systems seems to originate from the different crystal nucleus. A small crystal nucleus and large and thick LDH layered nanocrystals were developed for the nitrate system, whereas a large nucleus and relatively small and thin $\mathrm{LDH}$ layered nanocrystals were formed for the sulfate system.

The Stokes radius $\left(r_{\mathrm{s}}\right)$ and hydration radius $\left(r_{\mathrm{h}}\right)$ of the several anions are shown in Table 2. Although the $r_{\mathrm{s}}$ of monovalent anions $\left(\mathrm{NO}_{3}{ }^{-}\right.$, and $\left.\mathrm{Cl}^{-}\right)$is smaller than that of divalent anions $\left(\mathrm{SO}_{4}{ }^{2-}, \mathrm{CO}_{3}{ }^{2-}\right)$, their $r_{\mathrm{h}}$ has a slight difference. Only $\mathrm{NO}_{3}{ }^{-}$and $\mathrm{SO}_{4}{ }^{2-}$ were considered in this study. Under the same $r_{\mathrm{h}}$, a high charge number of anion will lead to high polarizability, that is, the polarizability of $\mathrm{SO}_{4}{ }^{2-}$ is larger than that of $\mathrm{NO}_{3}{ }^{-}$, indicating a relatively strong electrostatic bonding strength for $\mathrm{SO}_{4}{ }^{2-}$ as the interlayer species, whereas a weak electrostatic bonding strength for $\mathrm{NO}_{3}{ }^{-}$as the interlayer species within the LDH layered nanocrystals.

The metal salt used in LDH synthetic system is a strong electrolyte that dissociates completely. However, when the salt concentration is high, its degree of dissociation could not reach $100 \%$ due to the atmosphere of each ion with different charges. Some cations and anions would form an ion pair, which might

Table $2 \quad r_{\mathrm{s}}$ and $r_{\mathrm{h}}$ of several anions

\begin{tabular}{lcccc}
\hline Anions & $\mathrm{NO}_{3}{ }^{-}$ & $\mathrm{Cl}^{-}$ & $\mathrm{SO}_{4}{ }^{2-}$ & $\mathrm{CO}_{3}{ }^{2-}$ \\
\hline$r_{\mathrm{s}}(\mathrm{nm})$ & 0.129 & 0.121 & 0.230 & 0.266 \\
$r_{\mathrm{h}}(\mathrm{nm})$ & 0.335 & 0.332 & 0.379 & 0.394
\end{tabular}
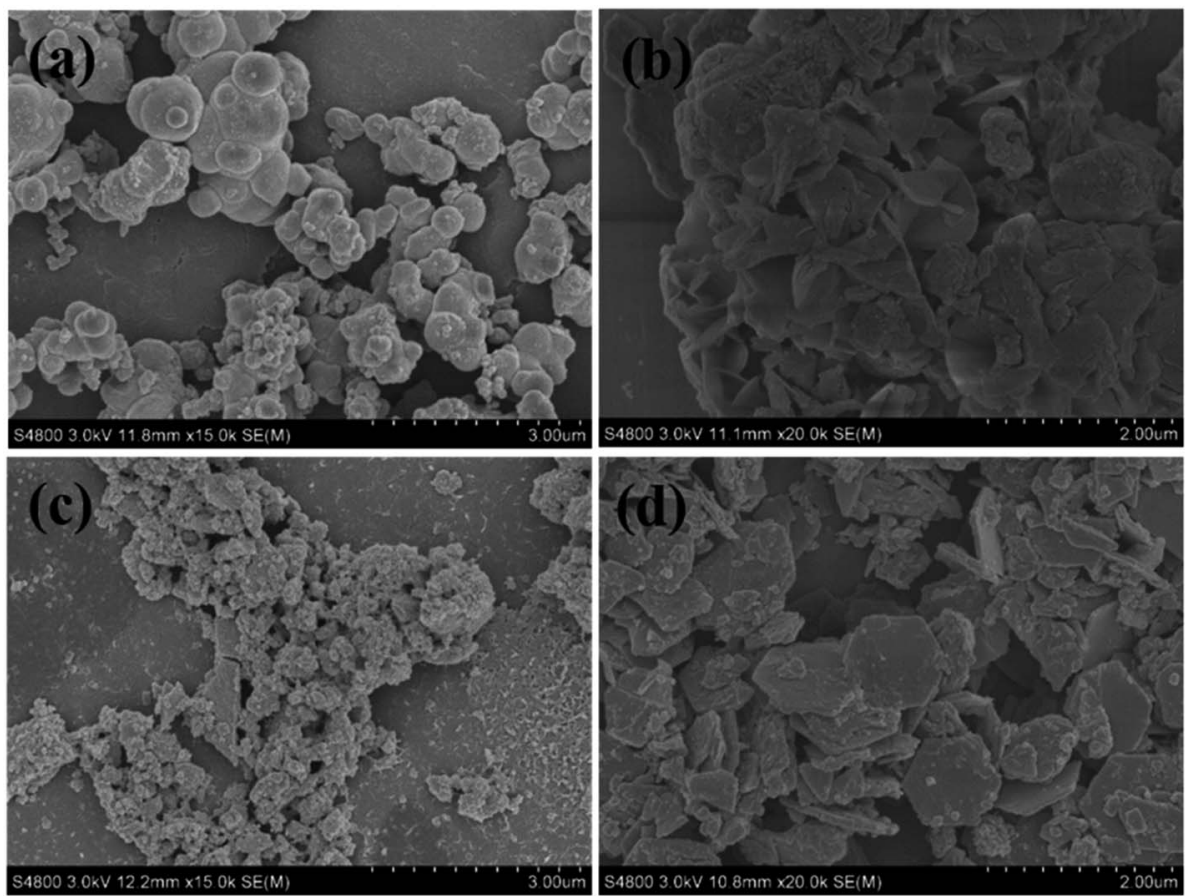

Fig. 3 FESEM pictures of LDH powder synthesized by (a and b) $\mathrm{MgAl}\left(\mathrm{SO}_{4}\right)$ and (c and d) $\mathrm{MgAl}\left(\mathrm{NO}_{3}\right)$ with different reaction times: (a, c) $3 \mathrm{~h}$; (b, d) $12 \mathrm{~h}$. 
influence the activity of ions in a solution, and even reduce the ability of ion function in a chemical reaction. Thus, the effective concentration, i.e., activity $(\gamma)$, of ions in chemical reaction, is lower than the apparent concentration. The ion strength $(I)$ must be considered in measuring the interaction between positive and negative ions. High concentration and high charge could lead to large $I$, strong restraint to each other and then small $\gamma$. If $I>10^{-4} \mathrm{~mol} \mathrm{~L}^{-1}$, then the restraint between ions would be strong and cannot be neglected. In this study, the initial concentrations $(c)$ of divalent and trivalent metal salts are 40 and $20 \mathrm{mmol} \mathrm{L}^{-1}$, respectively. After calculation, $I$ is $0.12 \mathrm{~mol} \mathrm{~L}^{-1}$ for $\mathrm{Mg}\left(\mathrm{NO}_{3}\right)_{2}$ and $0.12 \mathrm{~mol} \mathrm{~L}^{-1}$ for $\mathrm{Al}\left(\mathrm{NO}_{3}\right)_{3}$ in the nitrate system, whereas $I$ is $0.16 \mathrm{~mol} \mathrm{~L}^{-1}$ for $\mathrm{MgSO}_{4}$ and $0.15 \mathrm{~mol} \mathrm{~L}^{-1}$ for $\mathrm{Al}_{2}\left(\mathrm{SO}_{4}\right)_{3}$ in the sulfate system. All these values are higher than the critical $I$ of $10^{-4} \mathrm{~mol} \mathrm{~L}^{-1}$. In this case, the activity of the salt must be considered. $I$ of the sulfate is higher

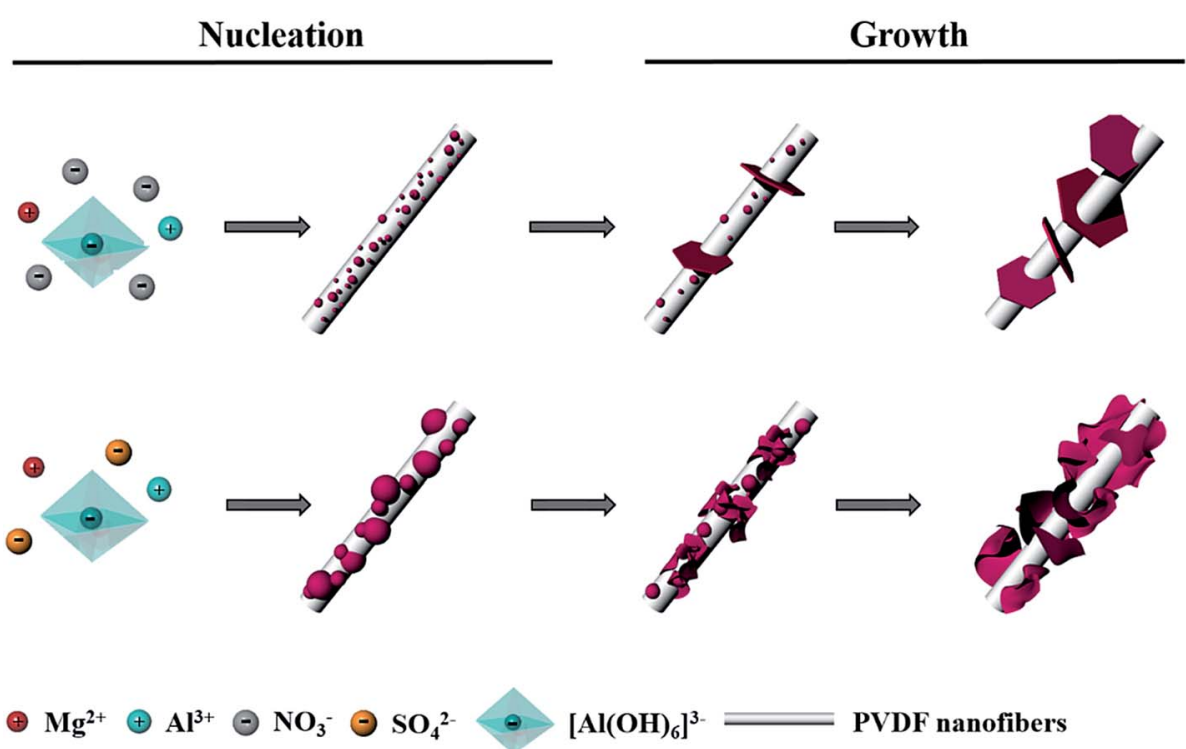

Scheme 1 Schematic illustration of the growth mechanism of LDHs on PVDF nanofiber surface in nitrate and sulfate system respectively.

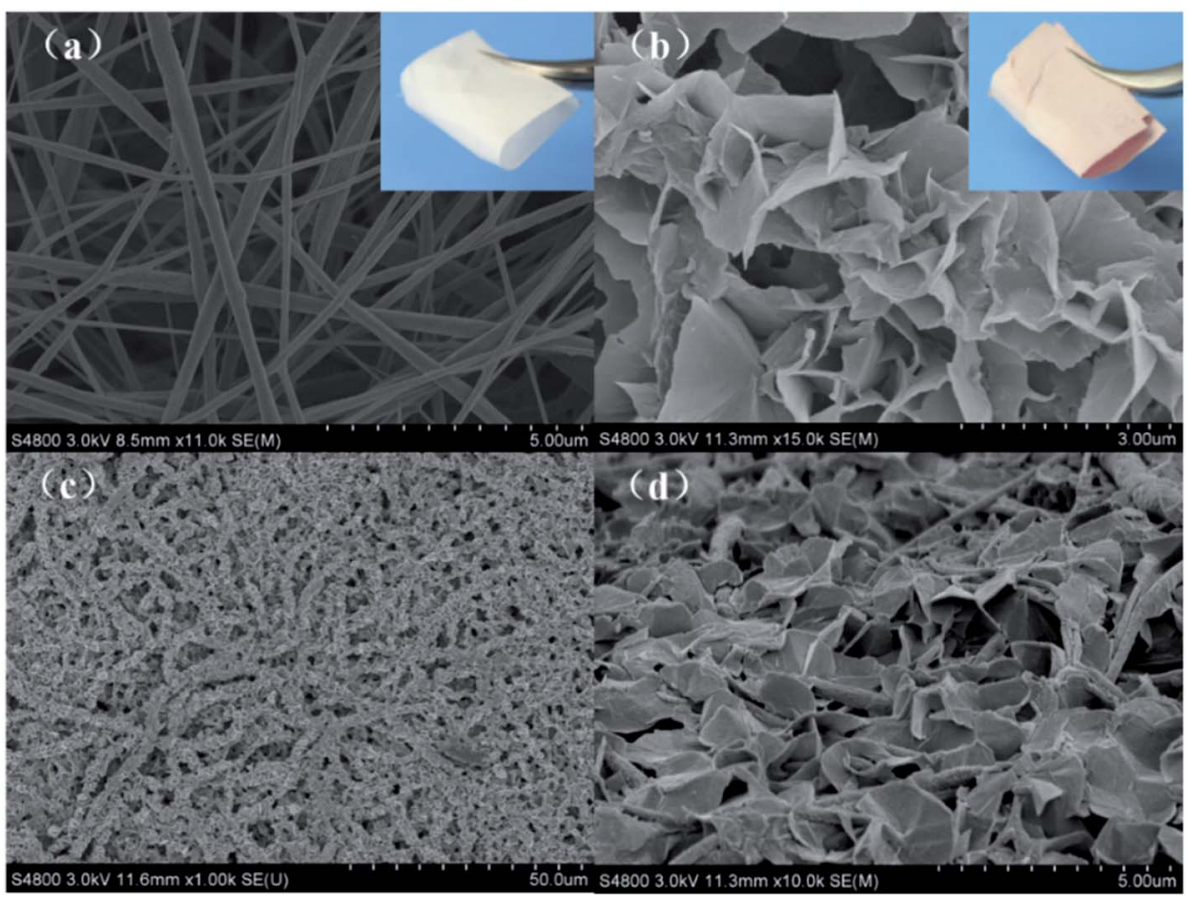

Fig. 4 FESEM images of (a) PVDF nanofibers; (b, c) PVDF@CoAl-LDH core/sheath fibers at different magnifications; (d) PVDF@CoAl-LDH core/ sheath fibers in the transverse direction of fibrous membrane. The insets in (a) and (b) are optical images of PVDF nanofibers and PVDF@CoAl$\mathrm{LDH}$ core/sheath fibers, respectively. 
than that of nitrate in the present case, implying $\gamma_{\text {nitrate }}>$ $\gamma_{\text {sulfate }}$. This finding indicates that the degree of dissociation of a sulfate in an aqueous solution is lower than that of a nitrate, that is, more effective ions exist in an aqueous solution of a nitrate system under the same molar concentration.

Then, the role of anions in forming LDH layered nanocrystals in a solution could be figured out. At a certain temperature, urea began to hydrolyze. The produced $\mathrm{OH}^{-}$ions immediately reacted with $\mathrm{Al}^{3+}$ to induce the anion coordination octahedrons (formulated as $\left[\mathrm{Al}(\mathrm{OH})_{6}\right]^{3-}$ in this study) because the precipitation $\mathrm{pH}$ of $\mathrm{Al}^{3+}$ was lower than that of $\mathrm{Mg}^{2+} .^{29}$ $\left[\mathrm{Al}(\mathrm{OH})_{6}\right]^{3-}$ subsequently reacted with $\mathrm{Al}^{3+}, \mathrm{Mg}^{2+}$, and anions to generate primary crystal nuclei. During coprecipitation, both homogeneous nucleation and growth processes compete to reduce the supersaturation of the solutes, and consequent $\mathrm{LDH}$ crystal sizes are dependent on the nucleation rates and their growth rates. ${ }^{27}$ As mentioned above, given that $\gamma_{\text {nitrate }}>\gamma_{\text {sulfate }}$ at the same initial molar concentration, a high activity could facilitate homogeneous nucleation and thus generate additional small nuclei. On the contrary, the growth rate of a primary crystal nucleus is dictated by the balance fluxes of ionic species between the attachment to and detachment from the primary nucleus surface. ${ }^{30}$ Under high temperature and high pressure, the former is dominant, whereas the latter is solely influenced by the bonding strength. The electrostatic bonding strength of $\mathrm{NO}_{3}{ }^{-}$is weaker than $\mathrm{SO}_{4}{ }^{2-}$, leading to higher fluxes of $\mathrm{NO}_{3}{ }^{-}$detaching from the nucleus surface. Therefore, the primary nuclei in the nitrate system have difficulty growing into large sizes despite of their large quantity. According to the classical nucleation and growth theory, after the spontaneous formation of nuclei, only those nuclei with sizes larger than a critical radius can grow up by the consumption of solutes followed by coarsening. ${ }^{30}$ Thus, although numerous crystal nuclei existed in a nitrate system, large nuclei which only account for a tiny proportion could continue to grow up. Subsequently, large and thick LDH layered nanocrystals were developed due to the lack of steric hindrance. In contrast, the crystal nuclei in the sulfate system are large and almost all of them could grow up synchronously. The growth of LDH layered nanocrystals would restrict mutually due to the serious steric hindrance, and dense, relatively small and thin LDH layered nanocrystals were developed ultimately.

Due to the weak interaction between LDHs and PVDF nanofiber surface, the nucleation and growth of LDH crystals on the nanofiber surface are identical to those in the solution. A great deal of small nuclei were formed on the surface of the PVDF nanofibers at the earlier stage and then only a small proportion with large size was allowed to grow up to welldefined hexagonal LDH layered nanocrystals with a large thickness in the nitrate system as shown in Scheme 1. However, in the sulfate system, large crystal nuclei were generated on the PVDF nanofiber surface first and then most of them could grow
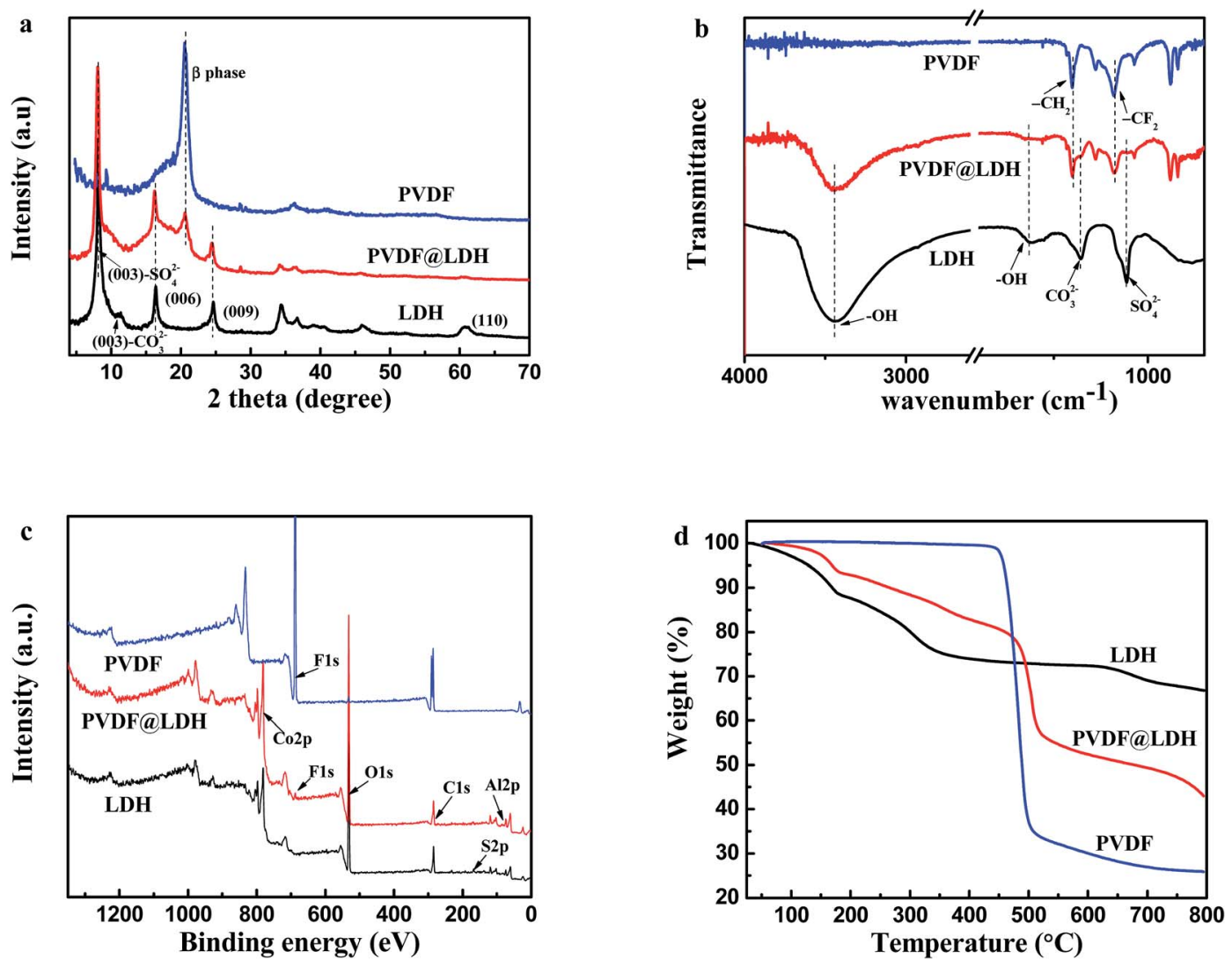

Fig. 5 (a) XRD patterns; (b) FT-IR spectra; (c) XPS spectra; (d) TGA profiles of LDH powder, PVDF nanofibers and PVDF@CoAl-LDH core/sheath fibers. 
up to thin and curly LDH layered nanocrystals owing to steric hindrance.

\section{Structure of PVDF@CoAl-LDH core/sheath fibers}

Given the application in dye removal, the PVDF@CoAl-LDH core/sheath fibers obtained from the sulfate system were selected as the research object and further characterized in detail. The FESEM image of the electrospun PVDF fibrous membrane exhibits a smooth surface with no beads and fiber diameter of about 200-400 nm (Fig. 4a). The PVDF membranes were flexible enough to be cut into appropriate sizes (the inset in Fig. 4a), and kept well even under high temperature and high pressure due to their intrinsic properties, i.e. excellent heat resistance and mechanical properties. ${ }^{22}$ The thickness of the membrane used in this experiment was about 50-60 $\mu \mathrm{m}$. Fig. $4 \mathrm{~b}$ presented the morphology of PVDF@CoAl-LDH core/sheath fibers at a high magnification, through which a lot of curled crystals with an average size of 1-2 $\mu \mathrm{m}$ can be clearly discerned. While at a low magnification (Fig. 4c), the as-obtained membrane shows a uniform and hierarchical structure varied from nano- to microscales. Fig. 4d shows the FESEM image of the PVDF@CoAl-LDH core/sheath fibers in the middle region of the transverse direction of the fibrous membrane. Numerous curly LDH layered nanocrystals grew tightly on the surface of the PVDF nanofibers, which confirmed that the growth of LDHs took place not only in the outside surface, but also in the inner region of the fibrous membrane. After the hydrothermal treatment, the fibrous membrane became pink from white as shown in the inset of Fig. 4 b, and was still kept with enough flexibility and mechanical strength.

Table 3 Surface elemental properties of LDH powder, PVDF nanofibers and PVDF@COAl-LDH core/sheath fibers

\begin{tabular}{|c|c|c|c|c|c|c|}
\hline & \multicolumn{2}{|l|}{ LDHs } & \multicolumn{3}{|c|}{ PVDF/CoAl-LDH } & \multirow{2}{*}{$\frac{\text { PVDF }}{\text { F 1s }}$} \\
\hline & Co $2 \mathrm{p}$ & AL 2p & Co $2 \mathrm{p}$ & $\mathrm{Al} 2 \mathrm{p}$ & F 1s & \\
\hline Peak BE (eV) & 781.5 & 74.1 & 781.76 & 73.86 & 688.01 & 688.00 \\
\hline Atomic (\%) & 12.66 & 6.02 & 14.39 & 7.43 & 1.23 & 52.38 \\
\hline
\end{tabular}

The typical XRD patterns of the LDH powder, PVDF nanofibers and PVDF@CoAl-LDH core/sheath fibers are shown in Fig. 5a. For pristine PVDF nanofibers, the strong diffraction peak at about $20.5^{\circ}$ is characteristic of the $\beta$-type crystal structure. ${ }^{31}$ The peaks of LDHs at $2 \theta$ values of $8.3^{\circ}(1.078 \mathrm{~nm}), 16.3^{\circ}$ $(0.544 \mathrm{~nm}), 24.6^{\circ}(0.362 \mathrm{~nm})$, and $60.8^{\circ}(0.152 \mathrm{~nm})$ can be indexed to (003), (006), (009), and (110) crystalline planes, respectively. The $d$-spacing of plane (003) is $1.078 \mathrm{~nm}$, which is consistent with the interplanar distance of $\mathrm{LDH}$ crystals with the interlayer anions of $\mathrm{SO}_{4}{ }^{2-}$, but the weak peak at $11.6^{\circ}$ presents the existence of a small amount of $\mathrm{CO}_{3}{ }^{2-}$ coming from the urea hydrolysis. ${ }^{32}$ Moreover, all characteristic peaks of PVDF and LDHs can be observed in the XRD pattern of PVDF@CoAlLDH core/sheath fibers, revealing the successful growth of LDHs on the surface of PVDF nanofibers. The results can also be supported by FT-IR spectra of these samples as shown in Fig. 5b. The peaks at $1400 \mathrm{~cm}^{-1}$ (deformation vibration of $-\mathrm{CH}_{2}$ ) and $1178 \mathrm{~cm}^{-1}$ (stretching vibration of $-\mathrm{CF}_{2}$ ) are considered as the characteristic peaks of PVDF. ${ }^{33}$ The broad peak centered at $3450 \mathrm{~cm}^{-1}$ is ascribed to the $\mathrm{O}-\mathrm{H}$ stretching vibration of interlayer water molecules and hydroxyl groups which exist in the LDH crystals. ${ }^{32}$

Compared with PVDF nanofibers, the $F$ signal strength of PVDF@CoAl-LDH core/sheath fibers was reduced significantly due to only 2-5 $\mathrm{nm}$ probing depth of the XPS technique (Fig. $5 \mathrm{c}$ ), implying that the surface of PVDF nanofibers was nearly fully covered by $\mathrm{LDH}$ layered nanocrystals. The $\mathrm{Co} / \mathrm{Al}$ atomic ratio (1.94) of the LDH crystals grown on the surface of PVDF

Table 4 Textural parameters of PVDF nanofibers and PVDF@CoAl$\mathrm{LDH}$ core/sheath fibers

\begin{tabular}{lll}
\hline & Samples & \\
\cline { 2 - 3 } & & $\begin{array}{l}\text { PVDF@CoAl-LDH } \\
\text { core/sheath fibers }\end{array}$ \\
Parameters & PVDF nanofibers & 12.14 \\
\hline Specific surface area $\left(\mathrm{m}^{2} \mathrm{~g}^{-1}\right)$ & 5.72 & 31.80 \\
Average pore diameter $(\mathrm{nm})$ & 26.86 & 0.0983 \\
Pore volume $\left(\mathrm{cm}^{3} \mathrm{~g}^{-1}\right)$ & 0.0384 &
\end{tabular}
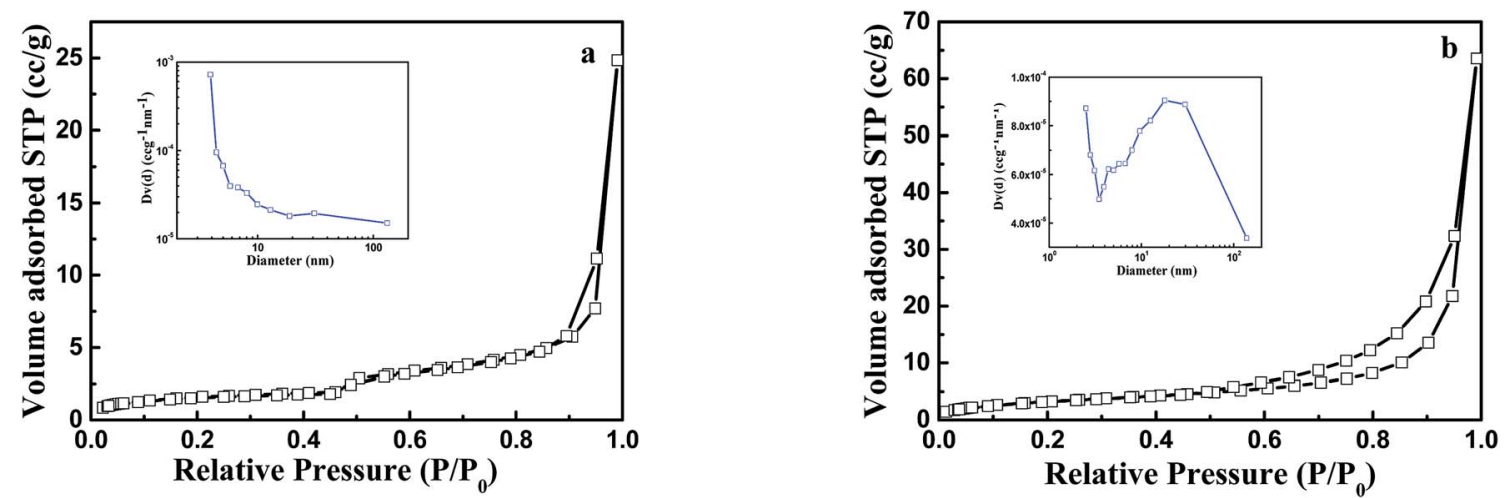

Fig. 6 Nitrogen adsorption-desorption isotherms of (a) PVDF nanofibers and (b) PVDF(CCoAl-LDH core/sheath fibers. The insets in (a) and (b) are the pore size distribution of the two samples. 
nanofibers is roughly equal to that (2.10) grown in the solution, both of them are similar to the stoichiometry ratio (2.0) (Table 3).

TGA tests were performed to further analyze the content of LDHs on the composite fibers. As shown in Fig. 5d, the decomposition temperature of PVDF nanofibers is in the range of $430-510{ }^{\circ} \mathrm{C}$ with a weight loss of $65 \%$. Three steps occurred during the calcination process of $\mathrm{LDH}$ powder with a total weight loss of $33.2 \%$, respectively corresponding to the removal of physically adsorbed water and interlayer water, the elimination of interlayer anions and the dehydroxylation of $\mathrm{LDH}$ layers. ${ }^{34}$ According to the thermal curve of PVDF@CoAl-LDH core/sheath fibers, the composition percentage of LDHs was calculated to be about $42 \%$, revealing a high mass loading of LDHs on the composite fibers, which is expected to be beneficial to a better MO adsorption performance.

Pore size of the as-obtained membranes was also investigated in this study. The nitrogen adsorption-desorption isotherms and the corresponding pore size distribution curves for PVDF nanofibers and PVDF@CoAl-LDH core/sheath fibers are displayed in Fig. 6. Both samples exhibit the typical isotherms of type IV with $\mathrm{H}_{3}$-type hysteresis loops, which are often attributed to the aggregates of plate-like particles giving rise to slit-shaped pores. ${ }^{35}$ The curves of pore size distribution indicate that the samples mainly consist of meso- and macropores, the latter of which can be further confirmed by the FESEM image in Fig. 4c. From the corresponding textural parameters presented in Table 4, PVDF@CoAl-LDH core/sheath fibers show a double increase of the specific surface area and a triple increase of the pore volume compared with the PVDF nanofibers, which may offer more active adsorption sites and improve the adsorption capacity of MO dye.

\section{Adsorption performance for MO}

Given their increased specific surface area and pore volume, PVDF@CoAl-LDH core/sheath fibers offer much more active adsorption sites and can improve the adsorption capacity of MO dye. The adsorption kinetics for MO are shown in Fig. 7a, in which the MO adsorption capacity increased rapidly in the first

Table 5 Kinetic parameters in the MO adsorption by PVDFaCoAl-LDH core/sheath fibers

\begin{tabular}{lclc}
\hline Pseudo-first-order model & \multicolumn{2}{c}{ Pseudo-second-order model } \\
\hline$q_{\mathrm{e}}\left(\mathrm{mg} \mathrm{g}^{-1}\right)$ & 248.60 & $q_{\mathrm{e}}\left(\mathrm{mg} \mathrm{g}^{-1}\right)$ & \\
$K_{1}\left(\times 10^{-3} \mathrm{~min}^{-1}\right)$ & 31.30 & $K_{2}\left(\times 10^{-3} \mathrm{~g} \mathrm{mg}^{-1} \mathrm{~min}^{-1}\right)$ & 273.76 \\
$R^{2}$ & 0.916 & $R^{2}$ & 0.17 \\
& & & 0.986
\end{tabular}
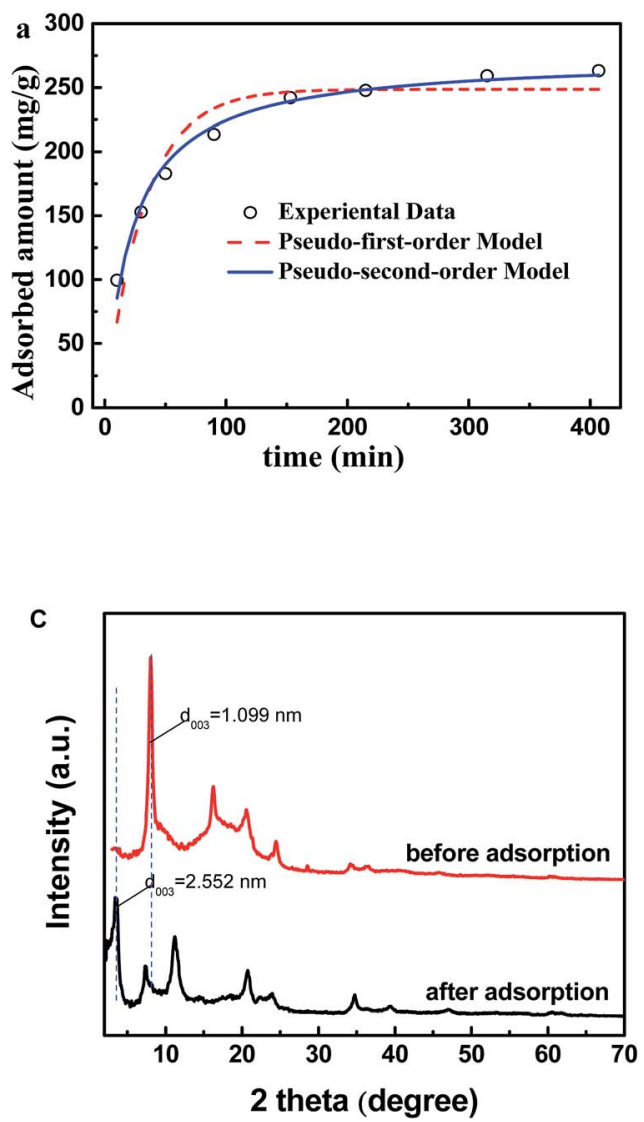
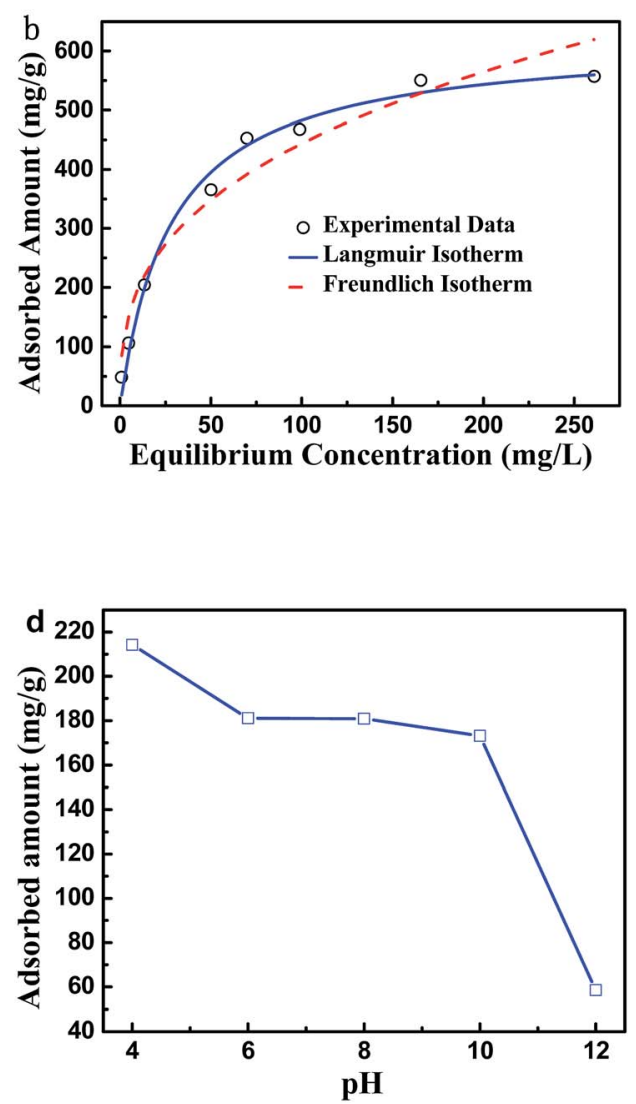

Fig. 7 (a) MO uptake by PVDF@COAl-LDH core/sheath fibers as a function of time at initial MO concentration of $200 \mathrm{mg} \mathrm{L}^{-1}$, the experimental data were fitted with pseudo-first-order and pseudo-second-order model; (b) adsorption isotherm of MO dye and the curve was fitted with Langmuir and Freundlich models; (c) XRD patterns of PVDF(CCOAl-LDH core/sheath fibers before and after MO adsorption; (d) MO adsorption property of PVDF@CoAl-LDH core/sheath fibers at different $\mathrm{pH}$. 
$50 \mathrm{~min}$, and then slowed down with time. When the contact time reached $400 \mathrm{~min}$, the MO adsorption capacity increased to $263 \mathrm{mg} \mathrm{g}^{-1}$. Equilibrium was not reached within the studied time, and the adsorption might continue at a slow speed after stopping the oscillation. Both pseudo-first-order and pseudosecond-order models were applied to describe the adsorption of MO dye on PVDF@CoAl-LDH core/sheath fibers. The calculated model parameters and linearization coefficients $\left(R^{2}\right)$ are

Table 6 Langmuir and Freundlich isotherms fitting parameters in the MO adsorption by PVDF@COAl-LDH core/sheath fibers

\begin{tabular}{lrlr}
\hline \multicolumn{2}{l}{ Langmuir isotherm } & \multicolumn{2}{l}{ Freundlich isotherm } \\
\hline$Q_{\mathrm{m}}$ & 621.17 & $K_{\mathrm{F}}$ & 89.193 \\
$K_{\mathrm{L}}$ & 0.0349 & $1 / n$ & 0.34835 \\
$R^{2}$ & 0.98955 & $R^{2}$ & 0.95365
\end{tabular}

tabulated in Table 5. The experimental data were fitted well with the pseudo-second-order model, implying the chemical adsorption characteristics.

The MO adsorption capability increased remarkably and then slightly with the MO concentration (Fig. 7b). The experimental data were fitted well with the two important isotherm models (Langmuir and Freundlich); the calculated model parameters and $R^{2}$ are given in Table 6. $R^{2}$ is 0.989 and 0.954 for Langmuir and Freundlich isotherm models respectively, indicating the dominance of the single layer adsorption mechanism of the system. The saturated adsorption capacity for MO is $621.17 \mathrm{mg} \mathrm{g}^{-1}$, which is higher than that for other previously reported fibrous adsorbents, such as polyaniline (skin)/ polyamide 6 (core) composite fibers $\left(58.7 \mathrm{mg} \mathrm{g}^{-1}\right),{ }^{36}$ hollow $\mathrm{CeO}_{2} / \mathrm{Al}_{2} \mathrm{O}_{3}$ fibers $\left(104 \mathrm{mg} \mathrm{g}^{-1}\right){ }^{37}$ and zwitterionic polyacrylonitrile fibers with hyperbranched polyethylenimine $\left(194 \mathrm{mg} \mathrm{g}^{-1}\right) .^{38}$
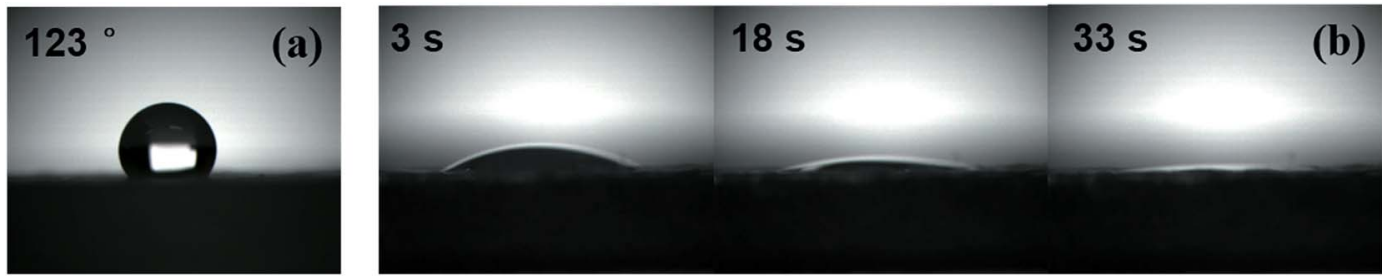

Fig. 8 Optical images of water contact angle on the surface of (a) PVDF nanofibers; (b) PVDFaCoAl-LDH core/sheath fibers.
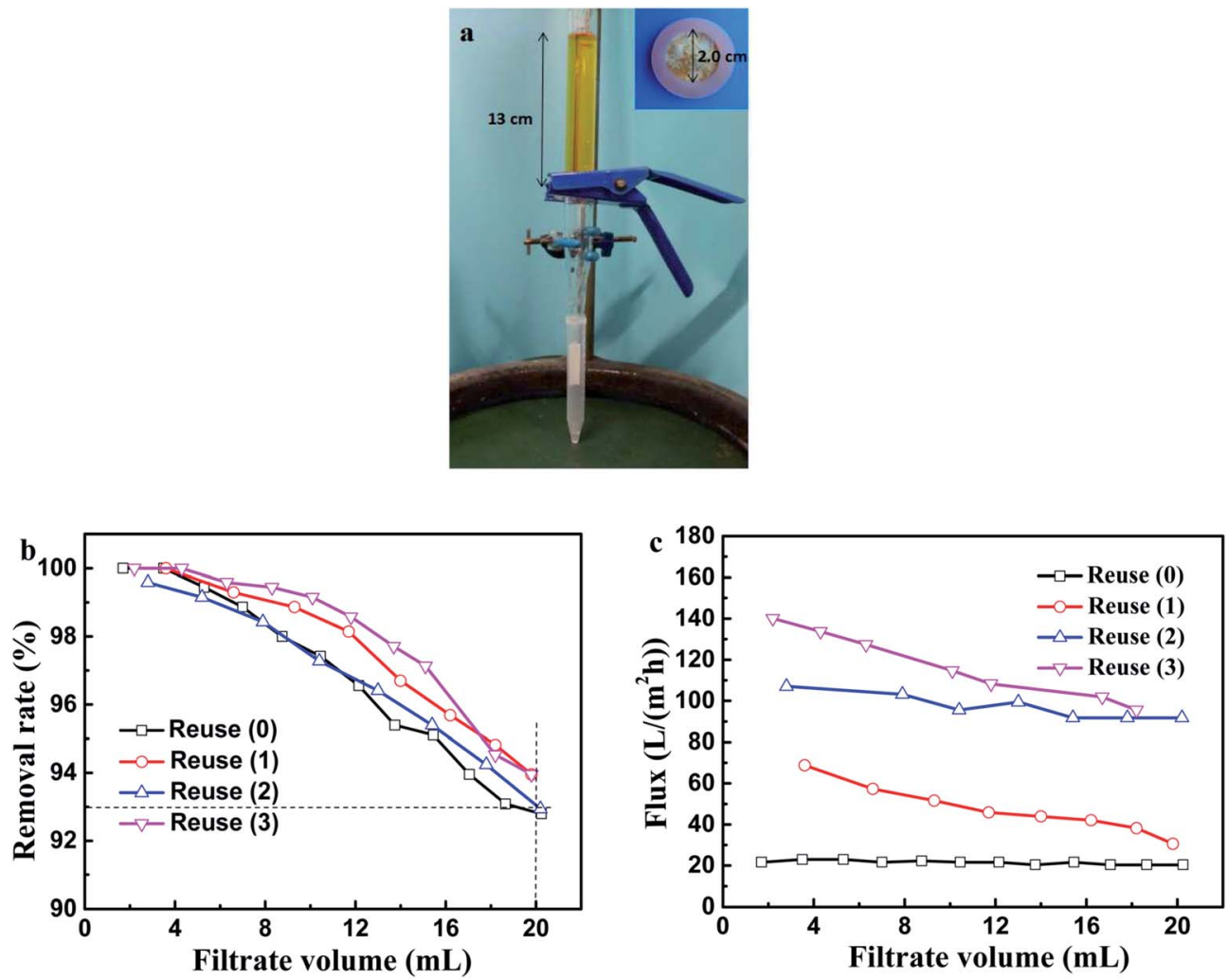

Fig. 9 (a) Optical image of the home made membrane filtration apparatus; (b) MO removal efficiency and (c) solution flux in different recycle runs by the treatment of PVDFaCoAl-LDH core/sheath fibers. 
XRD patterns of the PVDF@CoAl-LDH core/sheath fibers before and after MO adsorption were recorded to analyze the change of the LDH interlayer anions (Fig. 7c). The interplanar distance of LDHs increases from $1.099 \mathrm{~nm}$ to $2.552 \mathrm{~nm}$ after MO adsorption, which was ascribed to the intercalated MO anions. ${ }^{39}$ This finding meant that anion exchange existed between the interlayer anions and MO anions during adsorption process. Meanwhile the adsorption also could occur on the external surface of LDHs through the electrostatic attraction and hydrogen bonds, because it exhibited positive charge and contained a lot of hydroxyl groups. ${ }^{11}$ Thus the adsorption was both driven by the interlayer anion exchange and the external surface attraction. This conclusion can be further confirmed by studying the effect of $\mathrm{pH}$ on adsorption performance. As shown in Fig. 7d, the adsorption of MO kept at a high level with the $\mathrm{pH}$ value ranging from 4 to 10 . When $\mathrm{pH}$ was further increased to 12 , the adsorbed amount decreased precipitously to $58.55 \mathrm{mg}$ $\mathrm{g}^{-1}$. In a strong alkaline condition, the surface positive charge was reduced significantly by the adsorption of hydroxyl ions and the deprotonation of surface hydroxyl groups, which weakened the electrostatic interaction between the PVDF@CoAl-LDH core/sheath fibers and MO anions.

\section{Membrane filtration of MO solution and regeneration}

Hydrophilicity is important for membrane filtration of aqueous solution, which can be reflected directly by a water CA. As presented in Fig. 8, the CA of PVDF nanofibers was $123^{\circ}$ due to the intrinsic hydrophobic property of PVDF. While the CA of PVDF@CoAl-LDH core/sheath fibers was time-dependent and the water droplet could completely spread out and infiltrate along the membrane surface in a minute. The enhanced hydrophilicity was attributed to large amounts of hydroxyl on LDH surface, being beneficial for the membrane filtration properties.

When the dye solution passed through the membrane, the MO anions could be captured by the LDHs through electrostatic interaction and thus the polluted water was purified. Compared with static adsorption, filtration is fast and easy to be industrialized. The filtration apparatus and related experimental parameters are presented in Fig. 9a. The MO removal efficiency and solution flux along with the filtrate volume in different regeneration times are shown in Fig. $9 \mathrm{~b}$ and c, respectively. The MO removal rates were all kept above $93 \%$ in the first $20 \mathrm{~mL}$ of filtrate with nearly pure water attained, therefore exhibiting its high adsorption efficiency and excellent cycling stability. The flux became larger with the regeneration times increasing, which reached up to about $140 \mathrm{~L}\left(\mathrm{~m}^{2} \mathrm{~h}\right)^{-1}$ in the third recycle runs. This phenomenon might be caused by the loss of LDH powder deposited on the PVDF@CoAl-LDH core/sheath fibers during desorption, which rendered the diffusion channels accessible.

\section{Conclusion}

In summary, the morphology and loading amount of LDHs on PVDF fiber surface can be easily adjusted by varying the metal salt anions. In the nitrate system, small crystal nuclei could be formed on the surface of the PVDF nanofibers first and then only a small proportion with large sizes grew up to well-defined hexagonal LDH layered nanocrystals with large thicknesses. However, in the sulfate system, large crystal nuclei were generated first and most of them could grow up to thin and curly LDH layered nanocrystals owing to the serious steric hindrance. Therefore, well-defined PVDF@CoAl-LDH composite fibers with hierarchical structure and uniform core/sheath morphology could only be obtained in the sulfate system, which could act as an excellent adsorbent for treating dye (such as MO) solution. The adsorption of MO for the asobtained PVDF@CoAl-LDH core/sheath fibers is driven by the interlayer anion exchange and the external surface attraction. Meanwhile, the adsorption isotherm followed the single layer adsorption mechanism with the maximum adsorption capacity of $621.17 \mathrm{mg} \mathrm{g}^{-1}$. PVDF@CoAl-LDH core/sheath fibers also exhibit excellent adsorption performance and cycling stability when used as filtration membrane, and the MO removal rate of the membrane was still kept above $93 \%$ with the maximum flux of $140 \mathrm{~L}\left(\mathrm{~m}^{2} \mathrm{~h}\right)^{-1}$ despite three regeneration times. Therefore, the core/sheath composite fibers show high promise as adsorbent for the removal of MO due to their facile preparation, high efficiency, easy retrievability and cycling stability.

\section{Conflicts of interest}

There are no conflicts to declare.

\section{Acknowledgements}

This work was supported by the National Natural Science Foundation of China (Key Program, Grant 51333004 and G51673172).

\section{References}

1 M. T. Yagub, T. K. Sen, S. Afroze and H. M. Ang, Dye and its removal from aqueous solution by adsorption: A review, Adv. Colloid Interface Sci., 2014, 209, 172-184.

2 K. Singh and S. Arora, Removal of Synthetic Textile Dyes From Wastewaters: A Critical Review on Present Treatment Technologies, Crit. Rev. Environ. Sci. Technol., 2011, 41(9), 807-878.

3 H. Sun, L. Cao and L. Lu, Magnetite/reduced graphene oxide nanocomposites: one step solvothermal synthesis and use as a novel platform for removal of dye pollutants, Nano Res., 2011, 4(6), 550-562.

4 M. T. de Souza, E. Ambrosio, C. A. de Almeida, T. K. de Souza Freitas, L. B. Santos, V. de Cinque Almeida and J. C. Garcia, The use of a natural coagulant (Opuntia ficus-indica) in the removal for organic materials of textile effluents, Environ. Monit. Assess., 2014, 186(8), 5261-5271.

5 A. Stolz, Basic and applied aspects in the microbial degradation of azo dyes, Appl. Microbiol. Biotechnol., 2001, 56(1-2), 69-80. 
6 C. Pei, G. Han, Y. Zhao, H. Zhao, B. Liu, L. Cheng, H. Yang and S. Liu, Superior adsorption performance for triphenylmethane dyes on 3D architectures assembled by $\mathrm{ZnO}$ nanosheets as thin as similar to $1.5 \mathrm{~nm}$, J. Hazard. Mater., 2016, 318, 732-741.

7 G. Huang, J. Yao, W. Pan and J. Wang, Industrial-scale application of the plunger flow electro-oxidation reactor in wastewater depth treatment, Environ. Sci. Pollut. Res. Int., 2016, 23(18), 18288-18295.

8 A. Yurtsever, Ö. Çınar and E. Sahinkaya, Treatment of textile wastewater using sequential sulfate-reducing anaerobic and sulfide-oxidizing aerobic membrane bioreactors, J. Membr. Sci., 2016, 511, 228-237.

9 W. Huang, X. Yu and D. Li, Adsorption removal of Congo red over flower-like porous microspheres derived from $\mathrm{Ni} / \mathrm{Al}$ layered double hydroxide, $R S C A d v$., 2015, 5(103), 8493784946.

10 C. Barriga, M. Gaitán, I. Pavlovic, M. A. Ulibarri, M. C. Hermosĩn and J. Cornejo, Hydrotalcites as sorbent for 2,4,6-trinitrophenol: influence of the layer composition and interlayer anion, J. Mater. Chem., 2002, 12(4), 1027-1034.

11 F. Ling, L. Fang, Y. Lu, J. Gao, F. Wu, M. Zhou and B. Hu, A novel CoFe layered double hydroxides adsorbent: high adsorption amount for methyl orange dye and fast removal of Cr(vi), Microporous Mesoporous Mater., 2016, 234, 230-238.

12 C. Li, M. Wei, D. G. Evans and X. Duan, Layered double hydroxide-based nanomaterials as highly efficient catalysts and adsorbents, Small, 2014, 10(22), 4469-4486.

13 Y. Lu, B. Jiang, L. Fang, F. Ling, J. Gao, F. Wu and X. Zhang, High performance NiFe layered double hydroxide for methyl orange dye and $\mathrm{Cr}(\mathrm{vI})$ adsorption, Chemosphere, 2016, 152, 415-422.

14 X. Ruan, Y. Chen, H. Chen, G. Qian and R. L. Frost, Sorption behavior of methyl orange from aqueous solution on organic matter and reduced graphene oxides modified $\mathrm{Ni}-\mathrm{Cr}$ layered double hydroxides, Chem. Eng. J., 2016, 297, 295-303.

$15 \mathrm{~W}$. Lv, M. Du, W. Ye and Q. Zheng, The formation mechanism of layered double hydroxide nanoscrolls by facile trinal-phase hydrothermal treatment and their adsorption properties, J. Mater. Chem. A, 2015, 3(46), 23395-23402.

16 Y. Zhao, M. Wei, J. Lu, Z. L. Wang and X. Duan, Biotemplated Hierarchical Nanostructure of Layered Double Hydroxides with Improved Photocatalysis Performance, ACS Nano, 2009, 3, 4009-4016.

17 P. S. Kumar, J. Sundaramurthy, S. Sundarrajan, V. J. Babu, G. Singh, S. I. Allakhverdiev and S. Ramakrishna, Hierarchical electrospun nanofibers for energy harvesting, production and environmental remediation, Energy Environ. Sci., 2014, 7(10), 3192-3222.

18 V. Thavasi, G. Singh and S. Ramakrishna, Electrospun nanofibers in energy and environmental applications, Energy Environ. Sci., 2008, 1(2), 205-221.

$19 \mathrm{~J}$. Wu, N. Wang, Y. Zhao and L. Jiang, Electrospinning of multilevel structured functional micro-/nanofibers and their applications, J. Mater. Chem. A, 2013, 1(25), 7290-7305.
20 Y. E. Miao, R. Wang, D. Chen, Z. Liu and T. Liu, Electrospun self-standing membrane of hierarchical $\mathrm{SiO}_{2} @$ @gammaAlOOH (boehmite) core/sheath fibers for water remediation, ACS Appl. Mater. Interfaces, 2012, 4(10), 53535359.

21 S. Patel and G. Hota, Iron oxide nanoparticle-immobilized PAN nanofibers: synthesis and adsorption studies, RSC Adv., 2016, 6(19), 15402-15414.

22 W. Zhang, Y. Zhang, R. Fan and R. Lewis, A facile $\mathrm{TiO}_{2} / \mathrm{PVDF}$ composite membrane synthesis and their application in water purification, J. Nanopart. Res., 2016, 18(1), 31-41.

23 G. S. Sailaja, P. Zhang, G. M. Anilkumar and T. Yamaguchi, Aniosotropically organized LDH on PVDF: a geometrically templated electrospun substrate for advanced anion conducting membranes, ACS Appl. Mater. Interfaces, 2015, 7(12), 6397-6401.

24 M. Adachi-Pagano, C. Forano and J.-P. Besse, Synthesis of Alrich hydrotalcite-like compounds by using the urea hydrolysis reaction-control of size and morphology, $J$. Mater. Chem., 2003, 13(8), 1988-1993.

25 P. Ding, Z. Li, Q. Wang, X. Zhang, S. Tang, N. Song and L. Shi, In situ growth of layered double hydroxide films under dynamic processes: Influence of metal cations, Mater. Lett., 2012, 77, 1-3.

26 K. Okamoto, N. Iyi and T. Sasaki, Factors affecting the crystal size of the MgAl-LDH (layered double hydroxide) prepared by using ammonia-releasing reagents, Appl. Clay Sci., 2007, 37(1-2), 23-31.

27 X. Sun and S. K. Dey, Insights into the synthesis of layered double hydroxide (LDH) nanoparticles: Part 2. Formation mechanisms of LDH, J. Colloid Interface Sci., 2015, 458, 160-168.

28 C. Ribeiro, V. Sencadas, J. L. G. Ribelles and S. LancerosMéndez, Influence of Processing Conditions on Polymorphism and Nanofiber Morphology of Electroactive Poly(vinylidene fluoride) Electrospun Membranes, Soft Mater., 2010, 8(3), 274-287.

29 Y. Yang, X. Zhao, Y. Zhu and F. Zhang, Transformation Mechanism of Magnesium and Aluminum Precursor Solution into Crystallites of Layered Double Hydroxide, Chem. Mater., 2012, 24(1), 81-87.

30 J. J. D. Yoreo and P. G. Vekilov, principles of crystal nucleation and growth, Biomineralization, 2003, 54, 57-93.

31 P. Martins, A. C. Lopes and S. Lanceros-Mendez, Electroactive phases of poly(vinylidene fluoride): determination, processing and applications, Prog. Polym. Sci., 2014, 39(4), 683-706.

32 S. Guo, C. Zhang, H. Peng, W. Wang and T. Liu, Structural characterization, thermal and mechanical properties of polyurethane/CoAl layered double hydroxide nanocomposites prepared via in situ polymerization, Compos. Sci. Technol., 2011, 71(6), 791-796.

33 A. Qin, X. Li, X. Zhao, D. Liu and C. He, Engineering a Highly Hydrophilic PVDF Membrane via Binding TiO(2) Nanoparticles and a PVA Layer onto a Membrane Surface, ACS Appl. Mater. Interfaces, 2015, 7(16), 8427-8436. 
34 F. Z. Mahjoubi, A. Khalidi, M. Abdennouri and N. Barka, M$\mathrm{Al}_{-} \mathrm{SO}_{4}$ layered double hydroxides $(\mathrm{M}=\mathrm{Zn}, \mathrm{Mg}$ or $\mathrm{Ni}$ ): synthesis, characterization and textile dyes removal efficiency, Desalin. Water Treat., 2015, 57(45), 21564-21576.

35 K. S. W. Sing, Reporting Physisorption Data For Gas Solid Systems - With Special Reference To The Determination Of Surface-Area And Porosity, Pure Appl. Chem., 1982, 54(11), 2201-2218.

36 Y. Xia, T. Li, J. Chen and C. Cai, Polyaniline (skin)/polyamide 6 (core) composite fiber: preparation, characterization and application as a dye adsorbent, Synth. Met., 2013, 175, 163169.

37 X.-h. Zhou, W.-m. Kang, W. Xu and B.-w. Cheng, Flexible hollow $\mathrm{CeO}_{2} / \mathrm{Al}_{2} \mathrm{O}_{3}$ fibers: preparation, characterization and dye adsorption efficiency, RSC Adv., 2015, 5(103), 8453584542.

38 Y. Fan, H. J. Liu, Y. Zhang and Y. Chen, Adsorption of anionic $\mathrm{MO}$ or cationic $\mathrm{MB}$ from $\mathrm{MO} / \mathrm{MB}$ mixture using polyacrylonitrile fiber hydrothermally treated with hyperbranched polyethylenimine, J. Hazard. Mater., 2015, 283, 321-328.

39 G. Darmograi, B. Prelot, A. Geneste, G. Martin-Gassin, F. Salles and J. Zajac, How Does Competition between Anionic Pollutants Affect Adsorption onto $\mathrm{Mg}-\mathrm{Al}$ Layered Double Hydroxide? Three Competition Schemes, J. Phys. Chem. C, 2016, 120(19), 10410-10418. 